\title{
Image Quality Assessment Using Edge Correlation
}

\author{
Jayesh Ruikar, Ashoke Sinha, and Saurabh Chaudhury
}

\begin{abstract}
In literature, oriented filters are used for low-level vision tasks. In this paper, we propose use of steerable Gaussian filter in image quality assessment. Human visual system is more sensitive to multidirectional edges present in natural images. The most degradation in image quality is caused due to its edges. In this work, an edge based metric termed as steerable Gaussian filtering (SGF) quality index is proposed as objective measure for image quality assessment. The performance of the proposed technique is evaluated over multiple databases. The experimental result shows that proposed method is more reliable and outperform the conventional image quality assessment method.
\end{abstract}

Keywords-Image quality assessment, image quality metric, steerable Gaussian filter, subjective assessment

\section{INTRODUCTION}

$\mathbf{I}^{\mathrm{r}}$ MAGE quality assessment (IQA) is an important area of research in order to ensure quality of experience $(\mathrm{QoE})$ in the process of image capturing, transmission, compression, encoding, decoding, reconstruction and also for subsequent improvement. The quality of the image can be measured in two ways; such as subjective assessment and objective assessment. In the subjective assessment method, a subject or the human spectators will rate the quality of the given image based on the scale provided by IQA investigator. Since it involves human spectators, it is time consuming, more expensive and unrealistic. On the other hand, the objective assessment method is based on a mathematical model which can evaluate the image quality automatically. The objective IQA methods are further classified in the three main categories: (a) Full reference (FR), (b) Reduced reference (RR), and (c) No reference (NR), depending on the availability of full, partial or no information about the original image respectively. In this work, FR-IQA technique is presented considering the full information of the original image is available at the receiving end.

In conventional FR-IQA techniques, such as, mean square error (MSE) and peak signal-to-noise ratio (PSNR) are computationally simpler. However, they are not correlating well with human perception and fails to predict image quality [1]-[3]. To overcome this drawback, many researchers are progressing in the direction of finding a robust FR-IQA algorithm. One of the widely used IQA metric, structural similarity (SSIM), that compares reference and test images based on the loss in the luminance, contrast and structure component [4]. However, it is independent of image resolution and the viewing condition, so a multi-scale representation of SSIM (MSSIM) has been proposed in the literature [5]. The

All the authors are with the Department of Electrical Engineering, Nationa Institute of Technology Silchar, Cachar, Assam, INDIA. Pin- 788010 (e-mail jayeshruikar@gmail.com; ashokesinha2001@yahoo.co.in; saurabh1971 @gmail.com). complex wavelet structural similarity (CWSSIM) index is the ${ }^{1}$ extension of the SSIM in the complex wavelet transform [6]. Some FR-IQA approach involves human visual system (HVS) modelling in which the goal is to identify the HVS component which shows close relationship with human perception. The PSNR-human visual system (P-HVS) [7] and PSNR-human visual system modified (P-HVS-M) [8] are the two common approaches presented in the literature which measure the imperceptibility in the image. However, there are some limitations in the low level vision and structural degradation which are taken into account and presented in the visualsignal-to-noise ratio (VSNR) [9]. VSNR is motivated by the HVS stimulus response for near-threshold and suprathreshold distortions present in the image. Other measures, such as, noise quality measure (NQM) [10] and information fidelity criteria (IFC) [11] also show competitive performance to evaluation of image quality. To get more information on full reference image quality assessment, about its limitation, challenges, existing work and future work, interested readers can use the references [12]-[17]. This may lead to finding the new image quality metric (IQM) or to improve the performance of the existing image quality metric (IQM). There are several challenges still remain to get the reliable FR IQA, such as, illumination change, viewpoint change, spatial orientation selectivity. So, it is necessary to find the image quality based on the features in terms of the arbitrary orientation.

In perspective of the above, this paper focuses on edge based similarity measure since edges act as a major human visual sensitivity factor and are present in multiple directions in the image. In the presented work, the main contribution is that steerable Gaussian filtering (SGF) technique is presented for assessment of the quality of the image. The SGF is used particularly to get edge information at different orientations and also to get more degree of freedom in selecting the edges in the image. The application of steerable filter in IQA is motivated by the characteristics of edges as given in [18] which are (i) Local regularity/ smoothness / continuity and local irregularity /oscillation/ discontinuity which are also orthogonal to each other. (ii) There also exists anisotropic structures in the image and the edges always stretch out in multiple directions.

The rest of the paper is organized as follow. Section II, gives a brief background on steerable Gaussian filtering. In Section III, proposed image quality assessment based on steerable Gaussian filter (SGF) is presented. Section IV, presents the validation of the proposed SGF IQA on popular image databases, and the results are tabulated to indicate the performance of the proposed technique. Finally, Section V gives the conclusion. 


\section{STEERABLE GAUSSIAN FILTER: THEORY}

Steerable filters are also known as derivative filters have been developed by Freeman and Adelson [19], whereas, multi-scale pyramidal architecture is first reported in [20]. A class of asymmetric oriented filters or wedge filters for local analysis is proposed in [21]. Steerable filters are more precise in feature detection for edges such as " $\mathrm{X}$ ", " $T$ ", " $L$ ", junction etc that are present in the image [22].

In order to consider multiple structures such as edges at multiple orientations, here in this work, authors have used steerable Gaussian filter. Spatial orientation selectivity as well as frequency selectivity, robustness to the change in illumination and viewpoint are the important features of the steerable Gaussian filter [23]. Steerable filtering provides an effective means of oriented information which can help to produce a description of the image information. Steerable filters are applied in all the area of image processing such as feature detection, segmentation, image de-noising, texture modelling [24], image registration, face recognition, etc.

Steerable Gaussian filter is a set of oriented basis filters and the response of the filter at an arbitrary orientation can be synthesized from a linear combination of the basis filters [19].

In Cartesian coordinate the Gaussian function is $G(x, y)=e^{-\left(x^{2}+y^{2}\right)}$ and its two first order basis filter at $0^{\circ}$ and $\pi / 2$ are

$$
\begin{aligned}
G_{1}^{0}= & \frac{\partial}{\partial x} G(x, y)=-2 x e^{-\left(x^{2}+y^{2}\right)} \\
& \quad \text { and } G_{1}^{\pi / 2}=\frac{\partial}{\partial y} G(x, y)=-2 y e^{-\left(x^{2}+y^{2}\right)}
\end{aligned}
$$

Freeman defines the necessary conditions for steerability: It says- the overall response of a filter from two or more oriented filters is the linear combination of the individual filter responses at any arbitrary orientation. Following the property, steerable filters can be synthesized as $G_{1}^{\theta}=\cos (\theta) G_{1}^{0}+\sin (\theta) G_{1}^{\pi / 2}$. The second order derivative of Gaussian is given by $\left(G_{2}^{\theta}\right)[19]$. Mathematically, $G_{2}^{\theta}$ can be written as

$$
G_{2}^{\theta}=k_{a}(\theta) G_{2 a}+k_{b}(\theta) G_{2 b}+k_{c}(\theta) G_{2 c}
$$

Where, $G_{2 a}, G_{2 b}, G_{2 c}$ are the basis function and $k_{a}(\theta), k_{b}(\theta), k_{c}(\theta)$ are the interpolation function. Each of these terms can be expressed as,

$$
\begin{aligned}
& G_{2 a}=0.9213\left(2 x^{2}-1\right) e^{-\left(x^{2}+y^{2}\right)} \\
& G_{2 a}=1.843 x y e^{-\left(x^{2}+y^{2}\right)} \\
& G_{2 c}=0.9213\left(2 y^{2}-1\right) e^{-\left(x^{2}+y^{2}\right)} \\
& k_{a}(\theta)=\cos ^{2}(\theta) \\
& k_{b}(\theta)=-2 \cos (\theta) \sin (\theta) \\
& k_{c}(\theta)=\sin ^{2}(\theta)
\end{aligned}
$$

Similarly, for higher order filters it can be given as $G_{\mathrm{n}}^{\theta}$, here $n$ denotes the order of the Gaussian derivative and $(\ldots)^{\theta}$ represent the rotational operator.

$$
G_{n}{ }^{\theta}=\sum_{i=1}^{n+1} k_{i n}(\theta) \cdot G_{n}(i-1) \pi /(n+1)
$$

Here, $k_{i n}(\theta)$ represent the interpolation basis function.

Mathematically interpolation basis function for the nth order Gaussain derivative is expressed as

$$
\begin{aligned}
k_{i 1}(\theta)= & \cos (\theta)-(i-1) \pi / 2, \quad i=1,2 \\
k_{i 2}(\theta)= & 1 / 3(1+2 \cos (2(\theta-(i-1) \pi / 3))), \\
i=1,2,3 & \\
k_{i 3}(\theta)= & 1 / 4(2 \cos (\theta-(i-1) \pi / 4)+ \\
& 2 \cos (3(\theta-(i-1) \pi / 4))), \quad i=1,2,3,4
\end{aligned}
$$

More detail information about the higher order steerable Gaussain filter and their interpolation basis is given in [19], [20].

\section{PROPOSED IMAGE QUALITY ASSESSMENT BASED ON STEERABLE GAUSSIAN FILTERING (SGF IQA)}

Let $I(x, y)$ denote the reference image and $D(x, y)$ is the distorted version of the reference image. At any point $(x, y)$ in the image plane, $G(\theta)$ denote the response of a steerable Gaussian filter at $\theta^{\circ}$ orientation. The response of the filter is obtained by the $2 \mathrm{D}$ convolution operation with the set of basis filters to an image. In this paper, we have used second order derivative of Gaussian ( $G_{2}^{\theta}$ ) from (2) to filter the original image $I(x, y)$ and distorted image $D(x, y)$. The filtered edge information on an original image at any specific orientation can be given by the convolution operation, $I(x, y) * G_{2}^{\theta}$ and represented as $F_{r e f}$. The filtered edge information for the distorted image after the convolution operation is $D(x, y) * G_{2}^{\theta}$ and denoted by $D_{\text {dist }}$. Here it can be seen that edge regularity is present along a particular direction whereas edge irregularity is present along the orthogonal direction. So in order to obtain the edge information, difference along the two orthogonal directions has been taken. In this paper, four directions are taken into consideration. These are $0^{\circ}, 45^{\circ}, 90^{\circ}$ and $135^{\circ}$. Now suppose, if normalized filter coefficients corresponding to $0^{\circ}, 45^{\circ}, 90^{\circ}$ and $135^{\circ}$ degrees are taken then from these orientations, we can note that $0^{\circ}, 45^{\circ}$ represents the edge regularity along the direction and $90^{\circ}, 135^{\circ}$ are the corresponding edge irregularity in orthogonal direction. So filtered edge information $\mathrm{F}$ can be obtained by taking the absolute difference of 


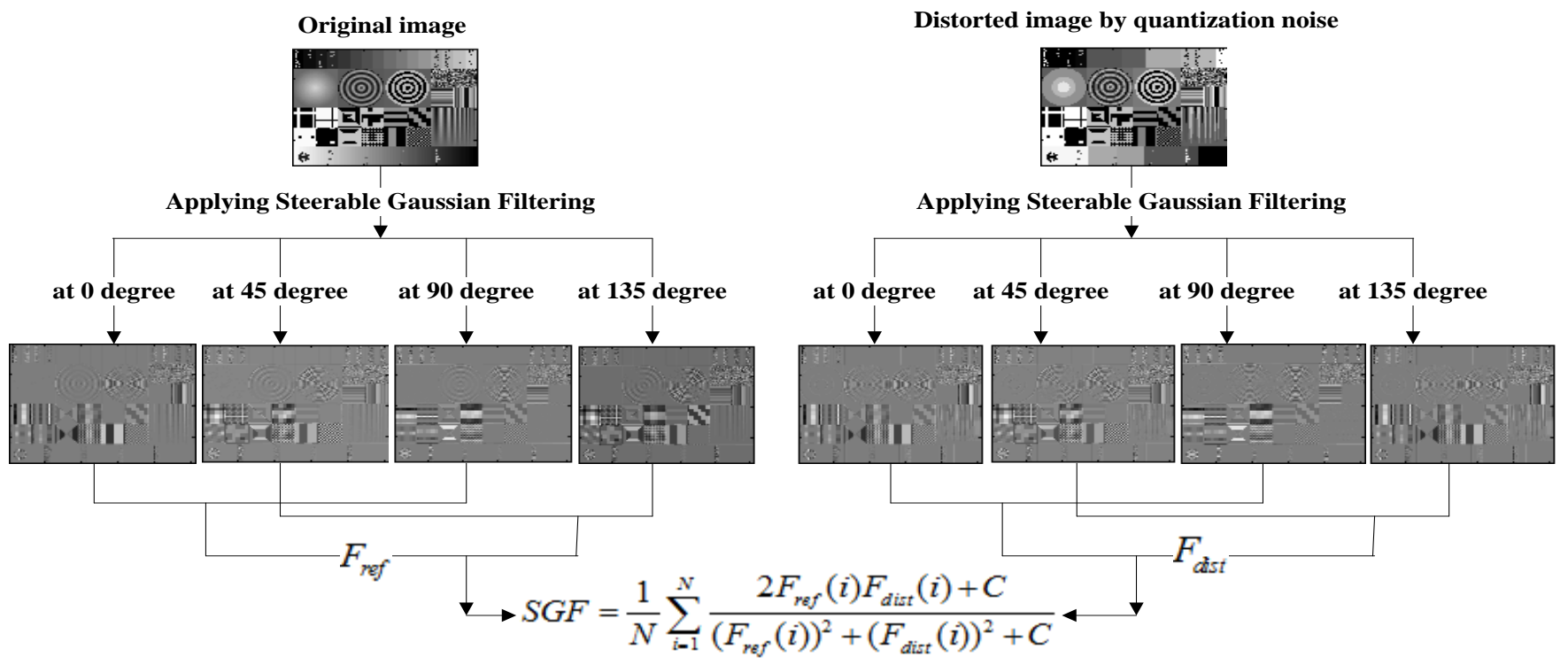

Fig. 1. General representation of SGF based Image Quality Assessment

$F($ at $\theta$ direction $)$ and $F\left(\right.$ at $\theta+\frac{\pi}{2}$ direction $)$. Thus,

by considering the direction of $0^{\circ}$ and $45^{\circ}$, $F$ (at $\theta$ direction) can be obtained and considering the direction of $90^{\circ}$ and $135^{\circ}, F($ at $\perp$ direction to $\theta)$ can be obtained. Mathematically,

$$
\begin{aligned}
& F(\text { at } \theta \text { direction })=\left|F^{0^{\circ}}-F^{90^{\circ}}\right| \\
& F\left(\text { at } \theta+\frac{\pi}{2} \text { direction }\right)=\left|F^{45^{\circ}}-F^{135^{\circ}}\right|
\end{aligned}
$$

For the reference image $I(x, y)$, edge information $F_{r e f}$ can be calculated as

$$
F_{\text {ref }}=\max \mid F(\text { at } \theta \text { direction })-F\left(\text { at } \theta+\frac{\pi}{2} \text { direction }\right) \mid
$$

Similarly for the distorted image $D(x, y)$, edge information $F_{\text {dist }}$ is given by

$$
F_{\text {dist }}=\max \mid F(\text { at } \theta \text { direction })-F\left(\text { at } \theta+\frac{\pi}{2} \text { direction }\right) \mid
$$

Here function $\max |. .$.$| represents the difference between the$ dual pair of directional edge information about the image.

So, based on similarity measurement between the edge regularity and edge irregularity, we propose image quality assessment metric based on steerable Gaussian filtering (SGF). It is defined as

$$
S G F=\frac{1}{N} \sum_{i=1}^{N} \frac{2 F_{r e f}(i) F_{\text {dist }}(i)+C}{\left(F_{r e f}(i)\right)^{2}+\left(F_{\text {dist }}(i)\right)^{2}+C}
$$

Where $i$ is the pixel index and $N$ is total number of pixel, $C$ is constant. The dynamic range of SGF quality index is $[0,1]$, where 0 represent the very low quality image and 1 represent the very good. The constant $\mathrm{C}$ can be used to scale the SGF score also helps to avoid denominator to become zero. Here, we have used $\mathrm{C}$ as 50 . Here, we have used orientation $0^{\circ}$ and $45^{\circ}$, i.e. $F$ (at $\theta$ direction) which gives us edge maps at that particular direction of the image. Considering the direction of $90^{\circ}$ and $135^{\circ}$, i.e. $F($ at $\perp$ direction to $\theta$ ) which also gives us edge maps of the direction. Now $F_{r e f}$ is the filtered edge information of reference image which can be obtained by the eq (9) and $F_{\text {dist }}$ is the filtered edge information of the distorted image obtained by the eq (10). The range of the $F_{r e f}$ and $F_{d i s t}$ is based on the image being used. The Quality measure of the image by the proposed SGF IQA is then calculated with the help of equation (11). It is seen that steerable Gaussian filter on the image oriented horizontally, vertically and diagonally gives the highest responses as there are vertical, horizontal and oblique edges are present in the image.

The general structure for evaluation of steerable Gaussian filtering (SGF) based Image quality assessment is depicted in Fig. 1. From the figure, we can see that the original image after passing through steerable Gaussian filter produce four different images in the direction of $0^{\circ}, 45^{\circ}, 90^{\circ}$ and $135^{\circ}$. These are essentially the edge maps in four different directions considered here which are also depicted in Fig. 1.

\section{RESULTS AND DISCUSSION}

The proposed SGF IQA technique has been validated by simulating over some of the images taken from three databases, namely, CSIQ database [25], IVC database [26] and TID2013 database [27], [28]. In this validation it is confirmed that the conventional measure like MSE fails to predict the 
quality of the image since it is not correlating well with the subjective assessment or the mean opinion score (MOS) score.

Zhou Wang et al. presented an experiment on the performance of MSE on the Lena image distorted by different types of distortions and the MOS score given by 22 observers[1]. In their experiment, they have considered MSE to grade the quality of the image. However, MSE is not a good measure to evaluate the quality since for identical MSE values there are difference in perceived quality of the image [1]. In [2] authors have shown that when there is degradation of the quality of the image varies linearly, there is a non-linear change in the MSE and PSNR. Here, in this paper an attempt has been made to evaluate the performance based on MSE on the images which are present in the CSIQ [25], IVC [26], and TID 2013 databases [27], [28] instead of evaluating performance based on MSE on Lena image which is outside the database [1]. Fig. 2 shows eight images from CSIQ database. Fig. 2(a), 2(c), 2(e) and 2(g) are the original images from CSIQ Databse whereas Fig. 2(b), 2(d), 2(f) and 2(h) are the distorted version of the Fig. 2(a), 2(c), 2(e) and 2(g). In the pair of images like 2(a) \& 2(b), 2(c) \& 2(d), 2(e) \& 2(f) and $2(\mathrm{~g}) \& 2(\mathrm{~h})$, MSE and SGF is calculated and their MOS is compared. The comparison of the MSE, SGF quality index and MOS is shown in Table 1. From Table we can see MSE values for the two comparison is same but MOS and SGF quality index are different. Similarly done the same experiment for the IVC [26], and TID 2013 databases [27], [28], which also shows that, there is a diverse performance of MSE with respect to MOS score. Thus we can conclude that MSE is not a correlated well with the human perception. The details of all the image names and their MOS, MSE and SGF based IQA technique for CSIQ [25], IVC [26], and TID 2013 databases [27], [28] are given in Table 1.

From the Table 1 it can be seen that there is a discrepancy not only in the MSE but also in the subjective experimentation. In the subjective experiments, the opinion of the particular subject or human is dependent on the mood, personal interest in the images. It can be seen that to analyse the images for the subjective evaluation, understanding of the image content is absolutely necessary. It is also essential to see whether the subjects are familiar with the images or not, and with the contents. In most of the psychophysical experiments, the person or subject is not familiar with the image, but as it is needed for the experimentation purpose, he or she has to perform it. For example, if the images are taken from an institute campus, and ask the subject or students from the campus to mark his or her opinion about the quality, he or she would interestingly rate the image with a somewhat more accurate opinion.

The performance of the proposed SGF IQA technique is now compared with other most popular image quality metrics. The criteria for such evaluation are Pearson linear correlation coefficient (PLCC or CC), Spearman rank order correlation coefficient (SROCC), Kendall's rank order correlation coefficient (KROCC) and root-mean-squared error (RMSE). To evaluate SROCC and KROCC, it is necessary to apply the regression method which provides non-linear mapping of MOS and the score computed from the IQM [30], [31]. The
CC and RMSE criteria help to define for prediction accuracy, whereas, KROCC and SROCC provides prediction monotonicity [30]. To evaluate CC, it is necessary to apply nonlinear regression between MOS and objective score. The CC, SROCC, and KROCC have their absolute values in the range 0 to 1 . RMSE measure provides the root mean square error between the MOS and objective score after nonlinear regression.

Generally, the score obtained from the objective IQA is fitted with the MOS Score. In the score obtained from the Objective IQA method may contain some non linearity. In order to account such non-linearity, a monotonic non linear function is used. In this paper, we have used a five parameter nonlinear monotonic logistic function given in [31] to fit the MOS and IQM score. This function expressed as

$$
f(x)=\beta_{1}+\left(\frac{1}{2}-\frac{1}{1+e^{\beta_{2}\left(x-\beta_{3}\right)}}\right)+\beta_{4} x+\beta_{5}
$$

Here $\beta_{1}, \beta_{2}, \beta_{3}, \beta_{4}$ and $\beta_{5}$ are the fitting parameters. The logistic function improve the correlation between MOS and the objective score.

The definition of CC, SROCC, KROCC and RMSE can be found in [29]. For the $n^{\text {th }}$ image in an image database, having objective score $p_{n}$ and subjective score $q_{n}$ (MOS or DMOS), the $\mathrm{CC}$ is defined as

$$
C C=\frac{\sum_{n=1}^{N}\left(p_{n}-\bar{p}\right) *\left(q_{n}-\bar{q}\right)}{\sqrt{\sum_{n=1}^{N}\left(p_{n}-\bar{p}\right)^{2} * \sum_{n=1}^{N}\left(q_{n}-\bar{q}\right)^{2}}}
$$

Spearman's rank order correlation coefficient (SROCC) is determined by

$$
\text { SROCC }=1-\frac{6 \sum_{n=1}^{N} d_{n}^{2}}{N\left(N^{2}-1\right)}
$$

Where $d_{n}$ is the difference between the nth image's rank in subjective and objective score. Again KROCC and RMSE is given by the following equation

$$
\begin{gathered}
\text { KROCC }=\frac{\text { no. of concordant pair- no. of discordant pairs }}{1 / 2 N(N-1)} \\
R M S E=\sqrt{\frac{1}{N} \sum\left(p_{n}-q_{n}\right)^{2}}
\end{gathered}
$$

Here, $N$ is the total number of images present. A better objective IQM measure should have higher CC, SROCC, and KROCC values, whereas it must have lower RMSE value. The evaluated prediction performance based on SROCC, KROCC, $\mathrm{CC}$, and RMSE across five popular IQA database is given in Table 2. The metrics which produces highest values of $\mathrm{CC}$, SROCC, KROCC and lowest RMSE are highlighted in boldface. 


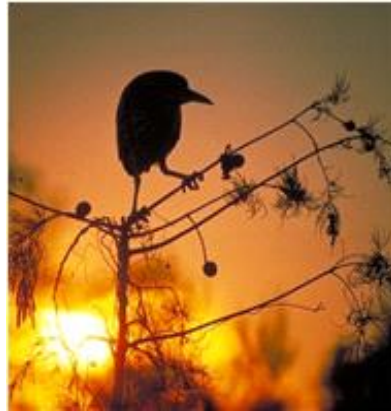

(a)

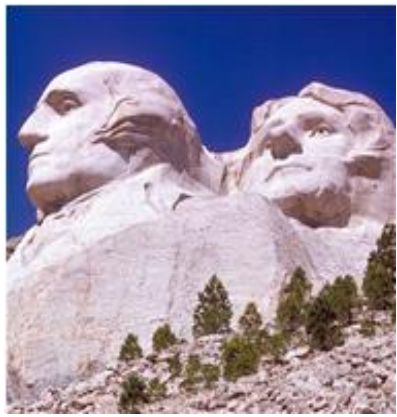

(e)

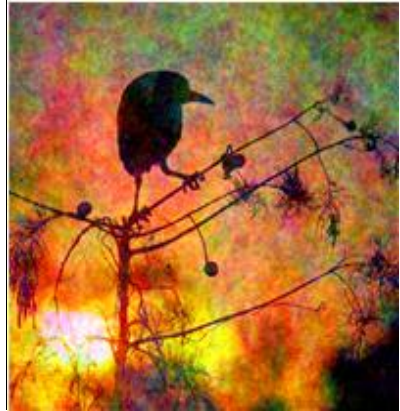

(b)

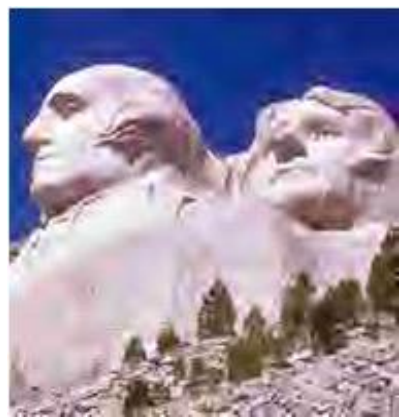

(f)

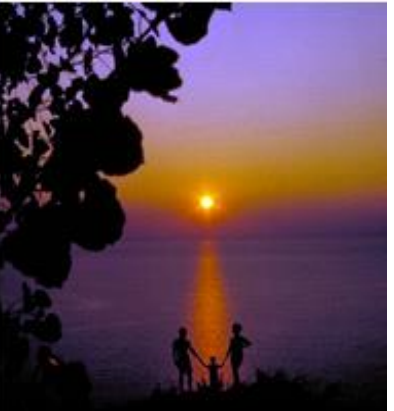

(c)

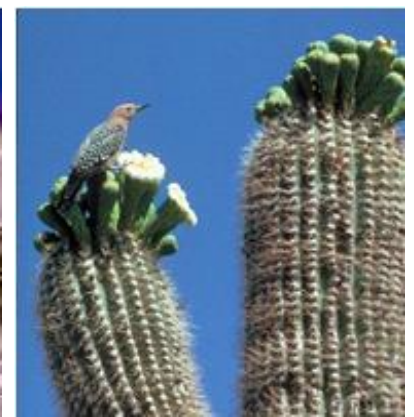

(g)

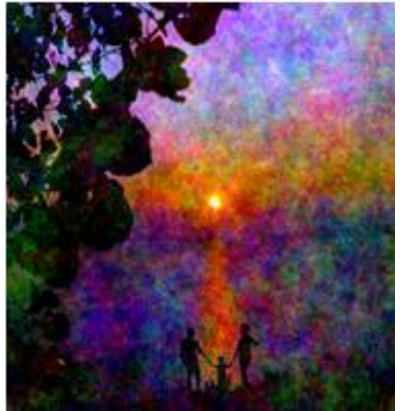

(d)

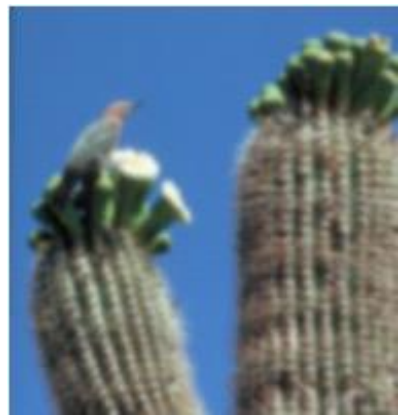

(h)

Fig. 2 Assessment of 8 images from CSIQ database[25] their MSE values are same but different MOS and SGF quality index by our approach (a) Original sunset sparrow image (b) is containing additive pink noise, (c) Original family image and (d) contain additive pink noise (e) original rushmore image (f) compressed by JPEG2000, (g) cactus image (h) blurred image

The performance of the proposed SGF IQA are evaluated over the five widely used IQA database such as MICT [32], LIVE [33], TID2008 [34], CSIQ [25] and TID2013 [27], [28]. Here, MICT database contains total 168 images, LIVE database consist of 982 images, TID2008 database is made up of 1700 images, CSIQ database is consist of 866 images whereas TID2013 database consist of 3000 images. The performance of the proposed SGF IQA is compared with the state-of-the-art techniques such as MSSIM [5], P-HVS [7], PHVS-M [8], SSIM [4],VSNR [9], UIQI [1], IFC[11], NQM [10], CWSSIM [6] and PSNR, MSE indices. From Table 2, it is observed that the performance of the proposed SGF based IQA technique over five benchmark databases outperforms all other techniques. In order to visualize the results scatter plots of MOS versus objective score after applying nonlinear regression are shown in Fig. 3 and Fig. 4. Fig. 3 shows the scatter plots of the subjective evaluation scores (MOS/DMOS) versus logistic method over TID 2013 database on the various IQA measures such as MSSIM [5], P-HVS [7], P-HVS-M [8], SSIM [4],VSNR [9], and UIQI [1].

Fig. 4 gives the scatter plots of the subjective evaluation scores (MOS/DMOS) versus logistic method over TID 2013 database on for other IQA such as IFC [11], NQM [10], CWSSIM [6], PSNR, MSE and proposed SGF IQA.

In the scatter plot of Fig. 3 and Fig. 4, each blue point corresponds to image quality measured by the objective approach versus quality obtained by the subjective approach of that image. These points are fitted by the nonlinear fitting with the help of equation (12). The scatter plot shows the close relationship between the subjective evaluation and the objective evaluation of each IQA. From the scatter plot of Fig. 4 (f), it can be seen that proposed SGF based IQA technique shows a better fitting of the curve thus indicating a better performance evaluation.

TABLE I

QUALITY ASSESSMENTS OF IMAGES FROM THE CSIQ, IVC AND TID 2013 DATABASES WITH DIFFERENT TYPES OF DISTORTIONS

\begin{tabular}{|c|c|c|c|c|c|c|}
\hline Database & Original image & Distorted image & Distortion added & MOS & SGF IQA & MSE \\
\hline \multirow{4}{*}{ CSIQ [25] } & Fig 2 (a) sunset_sparrow.png & Fig 2 (b) sunset_sparrow.fnoise.5.png & Pink noise & 0.7342 & 0.8202 & 332.0874 \\
\hline & Fig 2 (c) family.png & Fig 2 (d) family.fnoise.5.png & Pink noise & 0.6806 & 0.6820 & 332.2167 \\
\hline & Fig 2 (e) rushmore.png & Fig 2 (f) rushmore.jpeg2000.5.png & JPEG2000 & 0.7538 & 0.7482 & 631.4760 \\
\hline & Fig $2(\mathrm{~g})$ cactus.png & Fig 2 (h) cactus.BLUR.5.png & Blur & 0.7500 & 0.6725 & 631.8320 \\
\hline \multirow{4}{*}{ IVC [26] } & mandr.bmp & mandr_lar_r3.bmp & LAR coding & 4.0385 & 0.9361 & 232.4656 \\
\hline & barba.bmp & barba_jpeg_lumichr_r5.bmp & JPEG & 1.0769 & 0.8616 & 231.4743 \\
\hline & pimen.bmp & pimen_j2000_r5.bmp & JPEG2000 & 1.0000 & 0.7934 & 217.8015 \\
\hline & barba.bmp & barba_flou_f2.bmp & Blur & 3.3077 & 0.9778 & 217.2979 \\
\hline \multirow{4}{*}{$\begin{array}{l}\text { TID } 2013 \\
{[27],[28]}\end{array}$} & I05.bmp & i05_15_2.bmp & Block-wise distortions & 3.1000 & 0.9753 & 300.3883 \\
\hline & I03.bmp & i03_21_5.bmp & Lossy compression & 2.1000 & 0.8048 & 300.3287 \\
\hline & I09.bmp & i09_09_5.bmp & Image denoising & 2.5152 & 0.8285 & 286.0209 \\
\hline & I13.bmp & 13_05_4.bmp & JPEG2000 transmission errors & 4.1667 & 0.9799 & 286.0181 \\
\hline
\end{tabular}


TABLE II

COMPARISON OF THE PERFORMANCE OF THE PROPOSED SGF IQA OVER THE FIVE DATABASES

\begin{tabular}{|c|c|c|c|c|c|c|c|c|c|c|c|c|c|}
\hline Database & & MSSIM & PHVS & PHVSM & SSIM & VSNR & UQI & IFC & NQM & CWSSIM & PSNR & MSE & SGF \\
\hline \multirow{4}{*}{ MICT [32] } & SROCC & 907 & 0.8369 & 0.8813 & 0.8403 & 8608 & 0.7028 & 0.87 & 0.8871 & 0.7873 & 0.7221 & 0.7221 & 0.9141 \\
\hline & KROCC & 0.7316 & 0.6417 & 0.6975 & 0.6496 & 0.6745 & 0.5227 & 0.6745 & 0.7049 & 0.6026 & 0.5398 & 0.5398 & 0.7423 \\
\hline & $\mathrm{CC}$ & 0.9153 & 0.8335 & 0.8765 & 0.8474 & 0.8556 & 0.7164 & 0.6977 & 0.8795 & 0.7988 & 0.7325 & 0.5784 & 0.9275 \\
\hline & RMSE & 0.5316 & 0.7292 & 0.6355 & 0.7009 & 0.6478 & 0.8731 & 0.9457 & 0.5963 & 0.7941 & 0.8987 & 1.0768 & 0.4933 \\
\hline \multirow{4}{*}{ LIVE [33] } & SROCC & 0.5782 & 0.5525 & 0.5616 & 0.5431 & 0.5804 & 0.5425 & 0.5977 & 0.5804 & 0.5262 & 0.5049 & 0.5049 & 0.5988 \\
\hline & KROCC & 0.4491 & 0.4065 & 0.4142 & 0.4204 & 0.4339 & 0.4035 & 0.4478 & 0.4349 & 0.3843 & 0.3698 & 0.3698 & 0.4515 \\
\hline & $\mathrm{CC}$ & 0.6066 & 0.5953 & 0.6018 & 0.6243 & 0.6133 & 0.5807 & 0.6166 & 0.5964 & 0.563 & 0.5667 & 0.3883 & 0.6087 \\
\hline & RMSE & 12.805 & 12.941 & 12.863 & 12.582 & 12.783 & 13.175 & 12.68 & 12.99 & 13.311 & $\begin{array}{c}13.270 \\
3\end{array}$ & 14.8465 & 12.78 \\
\hline \multirow{4}{*}{$\begin{array}{c}\text { TID2008 } \\
{[34]}\end{array}$} & SROCC & 0.8542 & 0.5944 & 0.5612 & 0.6251 & 0.7045 & 0.5851 & 0.5675 & 0.6236 & 0.6453 & 0.5531 & 0.5531 & 0.8638 \\
\hline & KROCC & 0.6568 & 0.4764 & 0.4509 & 0.4528 & 534 & 0.4255 & 0.4236 & .46 & 0.4604 & 0.4027 & 0.4027 & 0.6733 \\
\hline & $\mathrm{CC}$ & 0.8451 & 0.5846 & 0.5528 & 0.6413 & 0.6818 & 0.6643 & 0.734 & 0.6127 & 0.6664 & 0.5734 & 0.5849 & 0.8734 \\
\hline & RMSE & 0.7173 & 1.0905 & 1.1183 & 1.0297 & 0.9813 & 1.0031 & 0.9114 & 1.0606 & 1.0005 & 1.0994 & 1.0884 & 0.6536 \\
\hline \multirow{4}{*}{ CSIQ [25] } & SROCC & 0.9039 & 0.8237 & 0.8169 & 0.8287 & 0.806 & 0.7986 & 0.7624 & 0.7374 & 0.7528 & 0.7991 & 0.7991 & 0.9173 \\
\hline & KROCC & 0.7343 & 0.6513 & 0.6503 & 0.6268 & 0.6227 & 0.6102 & 0.5853 & 0.5631 & 0.5546 & 0.6057 & 0.6057 & 0.7478 \\
\hline & $\mathrm{CC}$ & 0.8908 & 0.8209 & 0.7859 & 0.8097 & 0.7983 & 0.8173 & 0.8349 & 0.7447 & 0.7488 & 0.797 & 0.6395 & 0.9126 \\
\hline & RMSE & 0.1193 & 0.1499 & 0.1623 & 0.1541 & 0.1581 & 0.1513 & 0.1445 & 0.1752 & 0.174 & 0.1586 & 0.2018 & 0.1074 \\
\hline \multirow{4}{*}{$\begin{array}{l}\text { TID2013 } \\
\text { [27], [28] }\end{array}$} & SROCC & 0.7859 & 0.6533 & 0.6246 & 0.6274 & 0.6818 & 0.5507 & 0.5389 & 0.6465 & 0.6532 & 0.6394 & 0.6394 & 0.7882 \\
\hline & KROCC & 0.6047 & 0.5071 & 0.4814 & 0.4554 & 0.5084 & 0.3955 & 0.3939 & 0.4764 & 0.4713 & 0.4696 & 0.4696 & 0.6105 \\
\hline & $\mathrm{CC}$ & 0.8329 & 0.7178 & 0.682 & 0.6861 & 0.3039 & 0.6149 & 0.5519 & 0.677 & 0.7027 & 0.7017 & 0.676 & 0.8562 \\
\hline & RMSE & 0.6861 & 0.8631 & 0.9066 & 0.9019 & 1.3379 & 0.9776 & 1.0338 & 0.9124 & 0.882 & 0.8832 & 0.9135 & 0.6405 \\
\hline
\end{tabular}

\section{CONCLUSION}

This paper presents a new approach to full reference image quality assessment based on the steerable Gaussian filtering (SGF IQA) technique. The edges present in the natural images in multiple directions are taken as the key in the formulation of the model to assess the image quality. The performance of the proposed SGF IQA technique is validated and performance is compared. The results of the comparison show that the proposed technique is an effective method of evaluating the image quality with better performance.

\section{REFERENCES}

[1] Z. Wang and A. C. Bovik, "A universal image quality index," Signal Processing Letters, IEEE, vol. 9, no. 3. pp. 81-84, 2002.

[2] J. D. Ruikar, A. K. Sinha, and S. Chaudhury, "Review of Image Enhancement Techniques," in International Conference on Information Technology in Signal and Image Processing - itSIP, p. 2013.

[3] Q. Huynh-Thu and M. Ghanbari, "Scope of validity of PSNR in image/video quality assessment," Electronics Letters, vol. 44, no. 13. pp. 800-801, 2008.
[4] Z. Wang, A. C. Bovik, H. R. Sheikh, and E. P. Simoncelli, "Image quality assessment: from error visibility to structural similarity," IEEE Transactions on Image Processing, vol. 13, no. 4. pp. 600-612, 2004.

[5] Z. Wang, E. P. Simoncelli, and A. C. Bovik, "Multiscale structural similarity for image quality assessment," Signals, Systems and Computers, 2004. Conference Record of the Thirty-Seventh Asilomar Conference on, vol. 2. pp. 1398-1402 Vol.2, 2003.

[6] M. P. Sampat, Z. Wang, S. Gupta, A. C. Bovik, and M. K. Markey, "Complex Wavelet Structural Similarity: A New Image Similarity Index," IEEE Transactions on Image Processing, vol. 18, no. 11. pp. 2385-2401, 2009.

[7] K. Egiazarian, J. Astola, V. Lukin, F. Battisti, and M. Carli, "New full-reference quality metrics based on HVS," 2006.

[8] N. Ponomarenko, F. Silvestri, K. Egiazarian, M. Carli, J. Astola, and V. Lukin, "On between-coefficient contrast masking of DCT basis functions," in Proceedings of the Third International Workshop on Video Processing and Quality Metrics, 2007, vol. 4. 

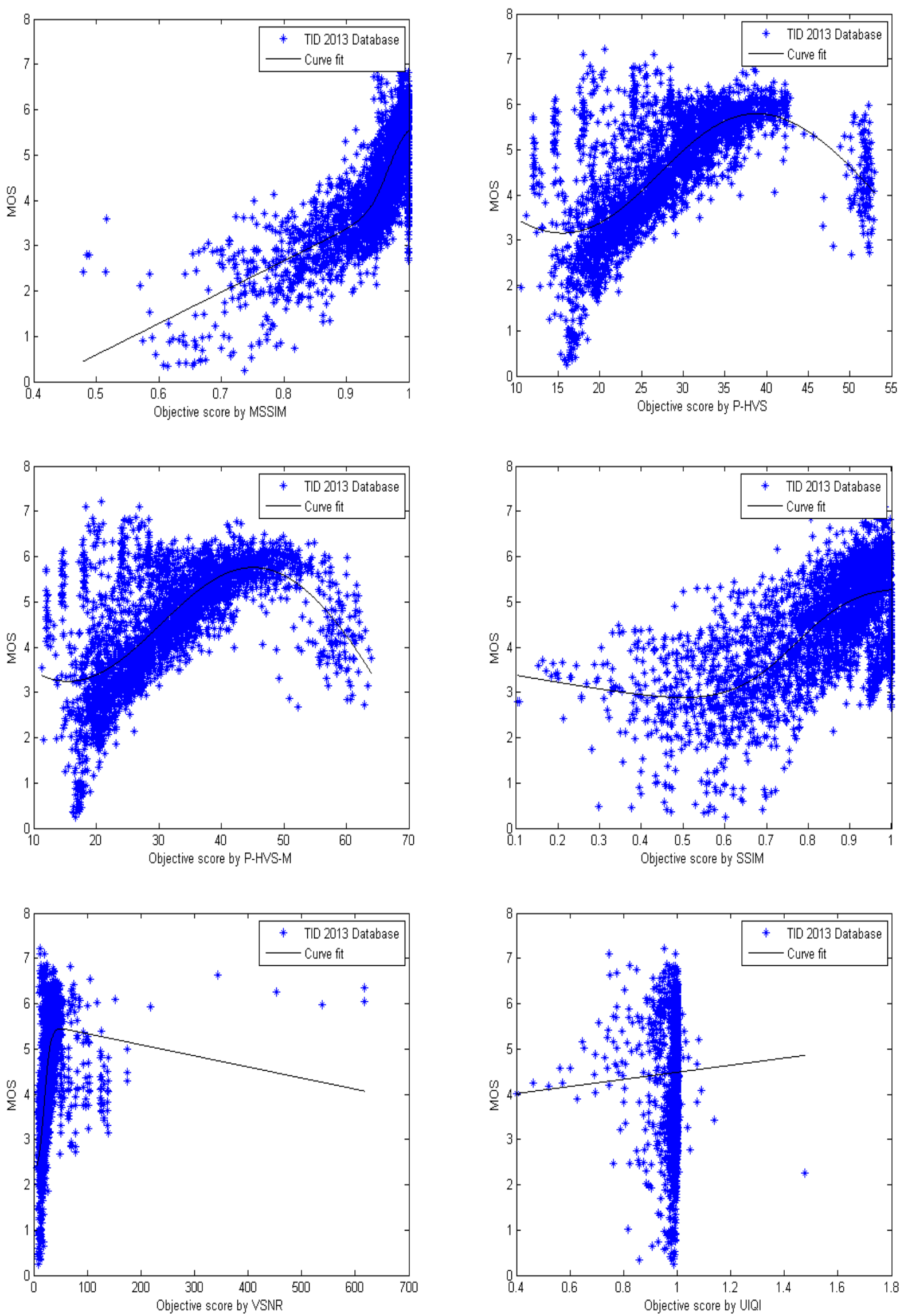

Fig. 3 Scatter plots of the subjective evaluation scores (MOS/DMOS) versus logistic method over the state-of-the-art IQA in TID 2013 database (a) MSSIM, (b) P-HVS, (c) P-HVS-M, (d) SSIM, (e) VSNR and (f)UIOI 

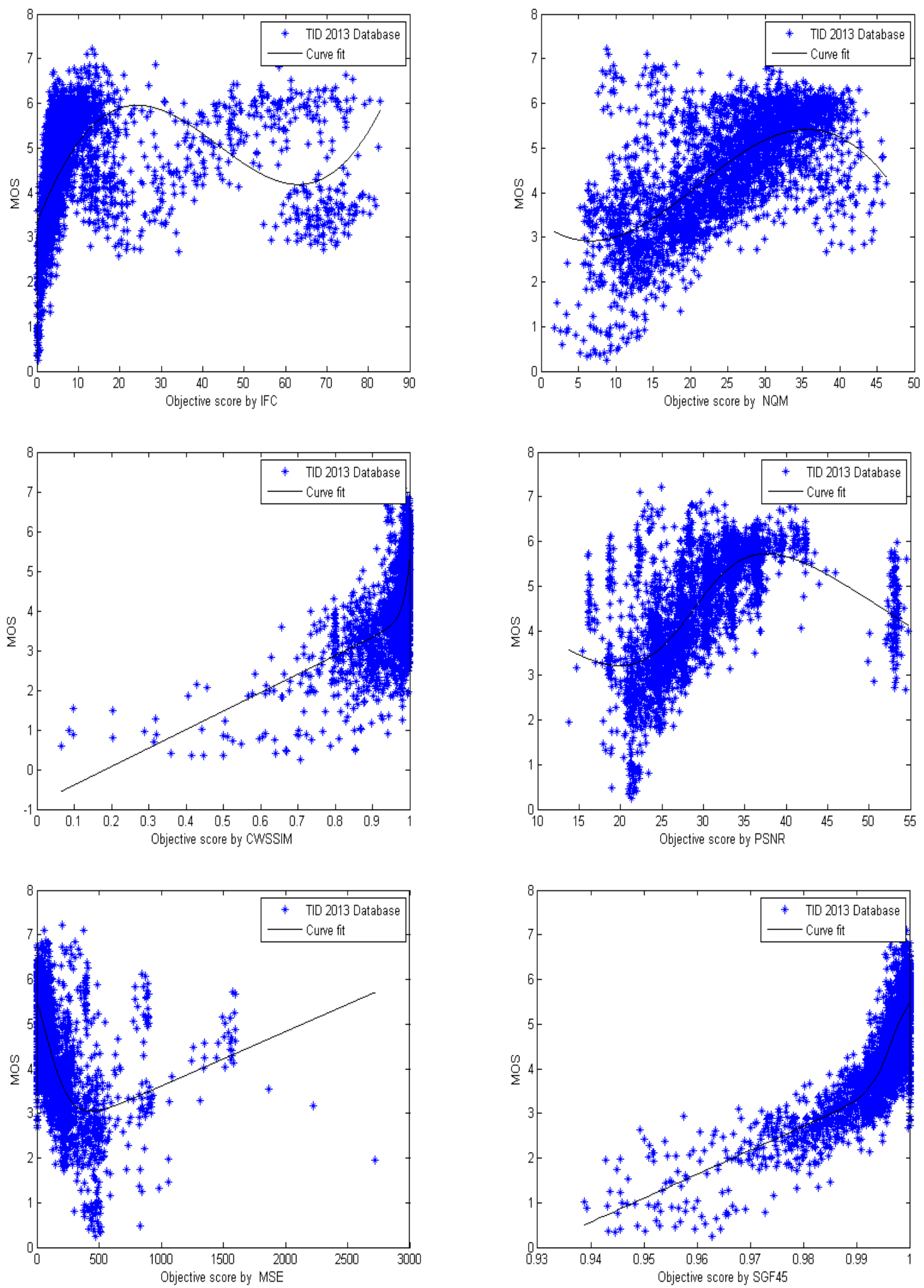

Fig. 4 Scatter plots of the subjective evaluation scores (MOS/DMOS) versus objective score) over the state-of-the-art IQA in TID 2013 database (a) IFC, (b) NQM, (c) CWSSIM, (d) PSNR (e) MSE and (f) proposed SGF IQA. 
[9] D. M. Chandler and S. S. Hemami, "VSNR: A Wavelet-Based Visual Signal-to-Noise Ratio for Natural Images," IEEE Transactions on Image Processing, vol. 16, no. 9. pp. 2284-2298, 2007.

[10] N. Damera-Venkata, T. D. Kite, W. S. Geisler, B. L. Evans, and A. C. Bovik, "Image quality assessment based on a degradation model," IEEE Transactions on Image Processing, vol. 9, no. 4. pp. 636-650, 2000.

[11] H. R. Sheikh, A. C. Bovik, and G. de Veciana, "An information fidelity criterion for image quality assessment using natural scene statistics," IEEE Transactions on Image Processing, vol. 14, no. 12. pp. 2117-2128, 2005.

[12] K. Seshadrinathan and A. Bovik, "Automatic prediction of perceptual quality of multimedia signals - a survey," Multimed. Tools Appl., vol. 51, no. 1, pp. 163-186, 2011.

[13] W. Lin and C.-C. Jay Kuo, "Perceptual visual quality metrics: A survey," J. Vis. Commun. Image Represent., vol. 22, no. 4, pp. 297-312, May 2011.

[14] F. Zhang, S. Li, L. Ma, and K. N. Ngan, "Limitation and challenges of image quality measurement," 2010, vol. 7744, pp. 774402-774408.

[15] D. M. Chandler, "Seven challenges in image quality assessment: past, present, and future research," ISRN Signal Process., vol. 2013, 2013.

[16] A. Moorthy and A. Bovik, "Visual quality assessment algorithms: what does the future hold?," Multimed. Tools Appl., vol. 51, no. 2, pp. 675-696, 2011.

[17] M. P. Eckert and A. P. Bradley, "Perceptual quality metrics applied to still image compression," Signal Processing, vol. 70, no. 3, pp. 177-200, Nov. 1998.

[18] X. Zhang, X. Feng, W. Wang, and W. Xue, "Edge strength similarity for image quality assessment," IEEE Signal Process. Lett., vol. 20, no. 4, pp. 319322, 2013.

[19] W. T. Freeman and E. H. Adelson, "The design and use of steerable filters," IEEE Transactions on Pattern Analysis and Machine Intelligence, vol. 13, no. 9. pp. 891-906, 1991.

[20] E. P. Simoncelli and W. T. Freeman, "The steerable pyramid: a flexible architecture for multi-scale derivative computation," International Conference on Image Processing, 1995. Proceedings.,, vol. 3. pp. 444-447 vol.3, 1995.

[21] E. P. Simoncelli and H. Farid, "Steerable wedge filters for local orientation analysis," Image Processing, IEEE Transactions on, vol. 5, no. 9. pp. 1377-1382, 1996.

[22] L. Jacques, L. Duval, C. Chaux, and G. Peyré, “A panorama on multiscale geometric representations, intertwining spatial, directional and frequency selectivity," Signal Processing, vol. 91, no. 12, pp. 2699-2730, Dec. 2011.
[23] J. J. Yokono and T. Poggio, "Oriented filters for object recognition: an empirical study," Sixth IEEE International Conference on Automatic Face and Gesture Recognition, 2004. Proceedings pp. 755-760, 2004.

[24] M. N. Do and M. Vetterli, "Rotation invariant texture characterization and retrieval using steerable waveletdomain hidden Markov models," Multimedia, IEEE Transactions on, vol. 4, no. 4. pp. 517-527, 2002.

[25] E. C. Larson and D. M. Chandler, "Categorical image quality (CSIQ) database," [Online] http://vision. okstate. edu/csiq, 2010.

[26] A. Ninassi, P. Le Callet, and F. Autrusseau, "Subjective quality assessment-IVC database," [online] http://www.irccyn.ec-nantes.fr/ivcdb/. 2006.

[27] N. Ponomarenko, O. Ieremeiev, V. Lukin, K. Egiazarian, L. Jin, J. Astola, B. Vozel, K. Chehdi, M. Carli, F. Battisti, and C.-C. J. Kuo, "Color image database TID2013: Peculiarities and preliminary results," Visual Information Processing (EUVIP), 2013 4th European Workshop on. pp. 106-111, 2013.

[28] N. Ponomarenko, O. Ieremeiev, V. Lukin, L. Jin, K. Egiazarian, J. Astola, B. Vozel, K. Chehdi, M. Carli, F. Battisti, and C.-C. J. Kuo, "A New Color Image Database TID2013: Innovations and Results," in Advanced Concepts for Intelligent Vision Systems SE - 36, vol. 8192, J. Blanc-Talon, A. Kasinski, W. Philips, D. Popescu, and P. Scheunders, Eds. Springer International Publishing, 2013, pp. 402-413.

[29] Z. Wang and Q. Li, "Information Content Weighting for Perceptual Image Quality Assessment," IEEE Transactions on Image Processing, vol. 20, no. 5. pp. 1185-1198, 2011.

[30] "Final Report From the Video Quality Experts Group on the Validation of Objective Models of Video Quality Assessment 2000.”VQEG.

[31] L. Zhang, D. Zhang, X. Mou, and D. Zhang, "FSIM: A Feature Similarity Index for Image Quality Assessment,", IEEE Transactions on Image Processing, vol. 20, no. 8. pp. 2378-2386, 2011.

[32] Y. Horita, K. Shibata, Y. Kawayoke, and Z. M. P. Sazzad, "MICT Image quality evaluation database." 2011.

[33] H. R. Sheikh, Z. Wang, L. Cormack, and A. C. Bovik, "LIVE image quality assessment database release 2." 2005.

[34] N. Ponomarenko and K. Egiazarian, "Tampere image database 2008 TID2008.” Available, 2009. 